\title{
Excimer laser for psoriasis treatment: A case report and short review
}

\author{
VALERIU ARDELEANU ${ }^{1-4^{*},}$ DIANA SABINA RADASCHIN ${ }^{4-6^{*}}$ and ALIN LAURENTIU TATU ${ }^{4-6 *}$ \\ ${ }^{1}$ Arestetic Plastic Surgery Clinic, 800098 Galați; ${ }^{2}$ Faculty of Medicine, Doctoral School, \\ Ovidius University, 900533 Constanța; ${ }^{3}$ Surgery Department, CFR General Hospital, 800223 Galați; \\ 4‘Dunărea de Jos' University, Clinical Department, 800008 Galați; ${ }^{5}$ Clinical Department of Dermatology, \\ Faculty of Medicine and Pharmacy, 'Dunărea de Jos' University, 800010 Galati; \\ ${ }^{6}$ St. Parascheva Infectious Diseases Clinical Hospital, 800179 Galați, Romania
}

Received December 13, 2019; Accepted January 16, 2020

DOI: $10.3892 /$ etm.2020.8529

\begin{abstract}
Psoriasis is a common cutaneous disease that has major implications in the quality of life of the patient. It is characterised by a chronic course and in some subtypes, it can be life threatening. Many studies have focused on the pathogenesis concerning this disease therefore improving the therapeutic approaches. Phototherapy represents a well-known treatment option for psoriasis. The depletion of T cells mostly from the epidermis, after exposure to phototherapy, validates the role of this treatment. The $308 \mathrm{~nm}$ excimer laser has the ability to treat mild, moderate and even severe but localised psoriasis plaques and plays an important role in the treatment management of psoriasis. In this report, we present the case of a patient diagnosed with plaque psoriasis, resistant to topical ointments, successfully treated with the $308 \mathrm{~nm}$ excimer laser. Although further studies are still required to consider the $308 \mathrm{~nm}$ excimer laser as a first line therapy, its high response rates have significantly influenced the therapeutic approach in psoriasis.
\end{abstract}

\section{Introduction}

Psoriasis is a common chronic cutaneous disease involving an abnormal inflammatory response characterised by an increase in proinflammatory cytokines consequential to the hyperproliferation of keratinocytes. Both environmental and genetic

Correspondence to: Dr Diana Sabina Radaschin, Clinical Department of Dermatology, Faculty of Medicine and Pharmacy, 'Dunărea de Jos' University, 1 Str. Albatrosului, 800010 Galati, Romania

E-mail: dianaradaschin@yahoo.com

\section{${ }^{*}$ Contributed equally}

Key words: excimer laser, psoriasis, lymphocytic apoptosis, decreased proliferation rate, UVB $308 \mathrm{~nm}$ factors interfere in the pathogenesis of psoriasis. The course of the disease usually involves a long period of cutaneous lesions which can result in psychological implications and severe impact on the quality of life.

The treatment based on the radiations of the UVB spectrum implies apoptosis of $\mathrm{T}$ lymphocytes and immunosuppressive effects resulting in clinical improvement of immune skin diseases. The $308 \mathrm{~nm}$ laser excimer represents a viable treatment option of inflammatory skin diseases such as vitiligo, atopic dermatitis, alopecia areata and psoriasis. It has been used in patients with psoriatic lesions that affect $<10 \%$ of the body area, recalcitrant plaques, or difficult to treat areas. Patients diagnosed with psoriasis may achieve clearance using the excimer laser technology more rapidly than using narrow-band UVB. Reduced number of sessions are necessary for treatment which signifies a lower cumulative dose than that in the UVB narrow band therapy.

Known as lepra graecorum in the ancient times, it was well described as a clinical condition in 1808 by the British physician Robert Willan. At present, due to many improvements in the research fields, the pathogenesis, genetical implications and risk factors are mainly known. Nearly 125 million people are suffering from psoriasis, currently, as shown by the International Federation of Psoriasis Associations (1). The data determined from the USA and European countries, suggest a prevalence estimated between 0.91 and $8.5 \%$ (2). Being a common disease, it is a subject of great interest in many fields of human research.

Early onset of psoriasis is associated with the expression of HLA-Cw6 (3-7), classified as type I psoriasis and is frequently seen in individuals younger than 40 years of age with positive family history of this disease. Type II psoriasis is associated with later onset, usually after the age of 40 (7).

The clinical appearance is divided into the following: the most common form is plaques psoriasis, which affects the majority of patients; another clinical presentation is guttate psoriasis which is associated with acute onset and is often detected in children after a streptococcal episode. The pustular form is characterised by amicrobial pustules and can affect different percentages of the body (localised or generalised). The life-threatening form of psoriasis is the erythrodermic 
form which consists of generalized erythema and suggests a low treatment compliance. The use of UVB therapy is indicated in the treatment of chronic plaques psoriasis and pustular psoriasis.

Concerning the anatomical body zones affected, psoriatic lesions can be observed on the scalp, palmoplantar sites, genital and nail sites. Usually, the nail involvement is observed in patients that often suffer from psoriatic arthritis as well $(3,8)$. Difficult to treat areas such as scalp, palmoplantar sites or auricular sites are easy to access using the excimer treatment. The ability to focus the radiation on the affected area represents an advantage of the excimer laser over the UVB narrow band.

Regarding the pathogenesis of psoriasis, many theories have been considered over time. The latest information concludes that an unknown trigger, on a genetically predisposed individual, starts a cascade of inflammatory and immune responses which lead to the formation of psoriatic plaques. Numerous immune cells have been found to have certain functions in the devilment of psoriatic lesions. Plasmacytoid dendritic cells production of interferon- $\alpha$, as well as tumor necrosis factor and interleukin 6 lead to the activation of dermal dendritic cells. These cells act as cell-presenting antigen to the naïve $\mathrm{T}$ cells in the lymph nodes. At that point, $\mathrm{T}$ cells differentiate into $\mathrm{T}$ helper 1 cells, $\mathrm{T}$ helper 17 and $\mathrm{T}$ helper 22 cells. The migration from the lymph nodes to the dermis through blood vessels, as well as lymphatics, is due to different cytokines secreted by keratinocytes. It promotes the formation of cutaneous lesions presented as psoriasis. The inflammatory response is maintained by the secretion of proinflammatory interleukins, nitric oxide radicals, vascular endothelial growth factor and angiopoietin which cause keratinocytic hyperproliferation and increased epidermal turnover. The understanding of these key elements leads to better therapeutic approaches $(3,4)$.

Another aspect fitting the pathogenesis of psoriasis is the more recent research based on studies on the cutaneous microbiome. The importance of Streptococcal upper respiratory tract infections are well known in the exacerbations of guttate psoriasis. It has been considered that a structural analogy between the K14 cytokeratin and the Streptococcal M protein is implicated in the formation of new guttate plaques (5). Additional link between plaque formation and the microorganisms that harbour our skin is demonstrated by the application of Malassezia ovalisto suspensions to the perilesional, unaffected, skin of an individual suffering from psoriasis. This led to the formation of new psoriatic lesions that improved after treatment with systemic antifungals (6).

The clinical appearance of psoriasis vulgaris consists of well demarcated erythematous scally plaques, with irregular outline, involving the elbows, knees, extensor surfaces and scalp. Annular or gyrate figures are present when the plaques coalesce, important characteristics which are valuable for the diagnosis of psoriasis on the scalp or genital region. The abundant white-silvery scales are easily removed, exposing small bleeding points (Auspitz sign) which correspond to the dilated blood vessels in the upper dermis (3).

Histologically, psoriasis is defined as epidermal thickening as a result of keratinocyte proliferation. The epidermal turnover is accelerated due to the releasing of multiple cytokines by immune cells, especially $\mathrm{T}$ lymphocytes. In lesional skin, dilated and elongated blood vessels are observed in the papillary dermis. Perivascular infiltrate is observed consisting mainly of lymphocytes and macrophages (3).

Lesion development in psoriatic patients involve a strong connection between the innate and the adaptive immune system. T helper cells are strongly involved in psoriatic pathogenesis. The secreted cytokines such as interleukin-17, interleukin-20, tumor necrosis factor- $\alpha$, have implications in maintaining the activation and hyperproliferation of keratinocytes, angiogenesis and chemoattractant for inflammatory cells such as neutrophils.

The cytotoxic role of the UVB treatments result in immunosuppressive effects achieving clinical improvement in psoriasis and in other inflammatory diseases.

\section{Case report}

We present the case of a 62-year old, male patient, which was referred to our clinic due to presence of widespread, sharply demarcated, erythematous plaques, with adherent silvery white scales on the surface of the lesions, irregularly shaped, ranging from 4 to $8 \mathrm{~cm}$, localised symmetrically on the elbows. The lesions had a tendency to coalesce into larger plaques (Fig. 1).

On the scalp region, the patient presented discrete erythematous scally patches, mainly in the temporal regions. The patient reported the onset of the lesions since childhood and has been in topical treatment with poor outcome. The treatment with excimer laser was proposed. The used dose regimen for each session was $400 \mathrm{~mJ} / \mathrm{cm}^{2}$ according to minimal erythema dose protocol. After 5 weeks, 1 session per week, the condition significantly improved (Fig. 2).

The study was approved by the Ethics Committee of the Romanian College of Physicians Galati County and the Aesthetic Plastic Surgery Clinic. Written informed consent was obtained from the patient prior to publication.

\section{Discussion}

Treatment options in psoriasis are divided in three main groups: topical therapy, systemic therapy and phototherapy. These therapies can be used separately or in combination. The choice is based upon the body surface affected by the disease and the patients' comorbidities.

The mechanism of action concerning phototherapy implies depletion of lymphocytes, especially those in the epidermis. It has been observed that after exposure to ultraviolet light, $\mathrm{T}$ helper 1 lymphocyte response shifts to $\mathrm{T}$ helper 2 response in lesional skin. The wavelength spectrum for psoriasis treatment ranges between 300 and $313 \mathrm{~nm}$ as shown in previous studies.

The use of 308-nm excimer laser has multiple purposes in dermatological diseases. It has been used in the treatment of vitiligo, alopecia areata, lichen planus, atopic dermatitis, eczema, prurigo and even mycosis fungoides $(9,10)$. In psoriasis it has shown efficiency in the treatment of recalcitrant plaques localised especially on the knees and elbows. It is indicated in moderate to severe psoriasis that affects $<10 \%$ of the body surface. Difficult to treat areas can be easily accessed 


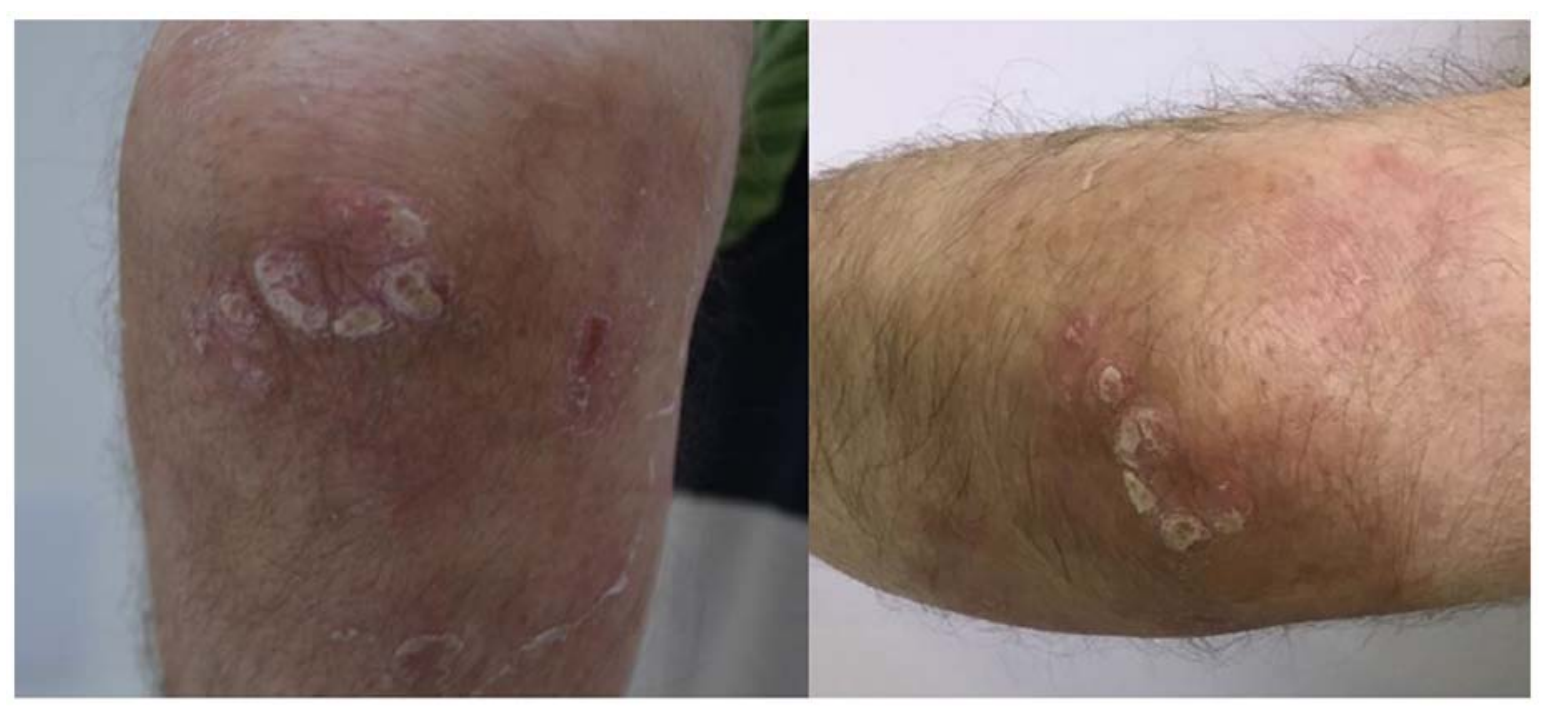

Figure 1. Plaque type psoriasis on the left elbow prior to treatment with excimer laser.

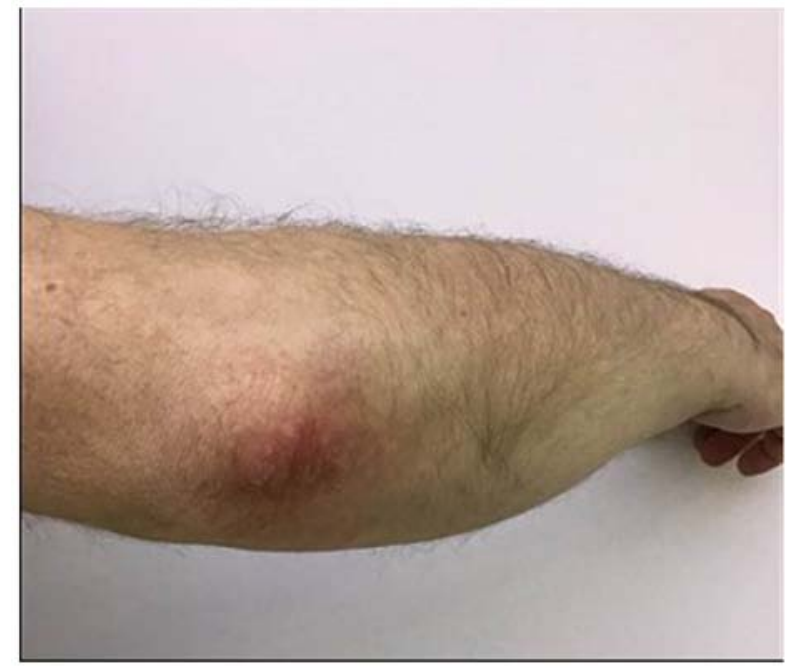

Figure 2. Plaque type psoriasis on the left elbow after 5 weeks of treatment with excimer laser.

using the laser therapy such as nose, ears and palpebral region that are hard to treat using conventional phototherapy. Other differences between phototherapy and excimer laser consists of lower UV exposer, decreased period of time of the treatment and the possibility to focus on the lesional skin, avoiding unaffected areas (11-13).

The 308-nm excimer laser induces lymphocytic apoptosis and decreases the proliferation rate of these cells obtaining a durable remission of the disease. Excimer derives from 'excited dimer' and is generated by a noble gas and halide (10-12). There are multiple protocols that evaluate the clinical response to treatment: the induration protocol, the minimal erythema dose and the minimal blistering dose.

The induration protocol refers to the treatment dosing. Taneja et al (14) used a primary dose regimen according to the induration of the plaques. Therefore, the dose regimen can be modified with the response to the treatment. The minimal erythema dose protocol implies testing the unaffected skin of the patient until an erythematous macule is obtained (15).
The minimal blistering dose regimen describes the minimal dose at which a blister is formed. The consecutive dosing regimens imply doses less powerful (16).

The PASI score improved more rapidly with the minimal blistering dose protocol, which implied clearance of the skin in a shorter period of time than the other protocols. The patient should be advised about the side effects and the formation of blisters $(15,16)$. The adverse reactions reported after treatment are moderate erythema, hypopigmentation, hyperpigmentation, and the formation of vesicles, depending on the protocols used $(10,11)$. Also, the adverse reactions of the medicines used to treat comorbidities and microbiome changes should be considered (17-25). Sometimes, if current available treatments for psoriasis fail or adverse reactions occur, complementary and alternative methods of treatment are available, psychological interventions and imaging techniques are occasionally needed (26-29).

The use of the 308-nm excimer for recalcitrant plaques of psoriasis laser represents a viable treatment alternative. The immunosuppression obtained by the 308-nm excimer laser can be explained by the lymphocytic apoptosis and the decreases of proliferation rate of these cells. In this way a durable remission of the disease can be obtained in a short period of time, illustrated by using the minimal blistering dose protocol.

The side effects of the 308-nm excimer such as erythema, blistering or hyperpigmentation occur on the body area treated despite using UVB narrow band. Regarding the cumulative doses of radiation, the 308-nm excimer laser therapy implies less radiation than UVB narrow band treatment. Compared to the UV phototherapy, the 308-nm excimer laser focus on the lesional skin and necessitate a lower exposure period.

In conclusion, the 308-nm excimer laser has been demonstrated as a practical therapeutic option for difficult to treat, resistant, psoriatic lesions. It is well tolerated, and the side effects are dose dependent. It can be used as monotherapy or combined with topical and systemic treatments. To establish the possibility that the excimer laser could become a first line treatment, further studies are required. 


\section{Acknowledgements}

Not applicable.

\section{Funding}

No funding was received.

\section{Availability of data and materials}

The datasets used and/or analyzed during the present study are available from the corresponding author on reasonable request.

\section{Authors' contributions}

The authors all had equal participation and equal rights to this article. VA examined, treated the subject and performed clinical photos, DSR and ALT were major contributors in writing the manuscript. VA, DSR and ALT were involved in all the stages of the study. All authors contributed to the conception and design of the study, as well as revising it. All authors read and approved the final manuscript to be published and agreed to be accountable for all aspects of the work in ensuring that questions related to the accuracy or integrity of any part of the work.

\section{Ethics approval and consent to participate}

The study was approved by the Ethics Committee of the Romanian College of Physicians Galati County and the Aesthetic Plastic Surgery Clinic. Written informed consent was obtained from the patient prior to publication.

\section{Patient consent for publication}

Written informed consent was obtained from the patient prior to publication.

\section{Competing interests}

The authors declare that they have no competing interests.

\section{References}

1. International Federation of Psoriasis Associations: World Psoriasis Day, 2015. https://ifpa-pso.com/our-actions/ world-psoriasis-day. Accessed June 27, 2019.

2. Griffiths CEM, van der Walt JM, Ashcroft DM, Flohr C, Naldi L, Nijsten T and Augustin M: The global state of psoriasis disease epidemiology: A workshop report. Br J Dermatol 177: e4-e7, 2017.

3. Bolognia J, Jorizzo JL and Schaffer JV (eds): Dermatology. Elsevier Saunders, Philadelphia, PA, 2012.

4. Ayala-Fontánez N, Soler DC and McCormick TS: Current knowledge on psoriasis and autoimmune diseases. Psoriasis (Auckl) 6: 7-32, 2016.

5. McFadden J, Valdimarsson H and Fry L: Cross-reactivity between streptococcal $\mathrm{M}$ surface antigen and human skin. Br J Dermatol 125: 443-447, 1991.

6. Narang T, Dogra S, Kaur I and Kanwar AJ: Malassezia and psoriasis: Koebner's phenomenon or direct causation? J Eur Acad Dermatol Venereol 21: 1111-1112, 2007.

7. Enerbäck C, Martinsson T, Inerot A, Wahlström J, Enlund F, Yhr M and Swanbeck G: Evidence that HLA-Cw6 determines early onset of psoriasis, obtained using sequence-specific primers (PCR-SSP). Acta Derm Venereol 77: 273-276, 1997.
8. Raychaudhuri SK, Maverakis E and Raychaudhuri SP: Diagnosis and classification of psoriasis. Autoimmun Rev 13: 490-495, 2014.

9. Spencer JM and Hadi SM: The excimer lasers. J Drugs Dermatol 3: 522-525, 2004

10. Mehraban S and Feily A: $308 \mathrm{~nm}$ excimer laser in dermatology. J Lasers Med Sci 5: 8-12, 2014.

11. Mavilia L, Mori M, Rossi R, Campolmi P, Puglisi Guerra A and Lotti T: $308 \mathrm{~nm}$ monochromatic excimer light in dermatology: Personal experience and review of the literature. G Ital Dermatol Venereol 143: 329-337, 2008.

12. Morita A, Weiss M and Maeda A: Recent developments in phototherapy: Treatment methods and devices. Recent Pat Inflamm Allergy Drug Discov 2: 105-108, 2008.

13. Abrouk M, Levin E, Brodsky M, Gandy JR, Nakamura M, Zhu TH, Farahnik B, Koo J and Bhutani T: Excimer laser for the treatment of psoriasis: safety, efficacy, and patient acceptability. Psoriasis (Auckl) 6: 165-173, 2016.

14. Taneja A, Trehan M and Taylor CR: 308-nm excimer laser for the treatment of psoriasis: Induration-based dosimetry. Arch Dermatol 139: 759-764, 2003.

15. Mudigonda T, Dabade TS and Feldman SR: A review of protocols for $308 \mathrm{~nm}$ excimer laser phototherapy in psoriasis. J Drugs Dermatol 11: 92-97, 2012.

16. Kemény L, Bónis B, Dobozy A, Bor Z, Szabó G and Ignácz F: 308-nm excimer laser therapy for psoriasis. Arch Dermatol 137: 95-96, 2001.

17. Tatu AL, Ciobotaru OR, Miulescu M, Buzia OD, Elisei AH, Mardarea N, Diaconu C, Robu S and Nwabudike LC: Hydrochlorothiazide: Chemical structure, therapeutic, phototoxic and carcinogenetic effects in dermatology Rev Chim 69: 2110-2114, 2018.

18. Nwabudike LC and Tatu AL: Response to - chronic exposure to tetracyclines and subsequent diagnosis for non-melanoma skin cancer in a large Mid-Western US population. J Eur Acad Dermatol Venereol 32: e159-e159, 2018

19. Tatu AL, Ionescu MA and Nwabudike LC: Contact allergy to topical momethasone furoate confirmed by rechallenge and patch test. Am J Ther 25: e497-e498, 2018.

20. Gheorghe I, Tatu AL, Lupu I, Thamer O, Cotar AI, Pircalabioru GG, Popa M, Cristea VC, Lazar V and Chifiriuc MC: Molecular characterization of virulence and resistance features in Staphylococcus aureus clinical strains isolated from cutaneous lesions in patients with drug adverse reactions. Rom Biotechnol Lett 22: 12321-12327, 2017.

21. Tatu AL and Nwabudike LC: Reply to: Kubiak K, et al: Endosymbiosis and its significance in dermatology. J Eur Acad Dermatol Venereol 32: e346-e347, 2018.

22. Tatu AL and Cristea VC: Pityriasis folliculorum of the back thoracic area: Pityrosporum, keratin plugs, or Demodex involved? J Cutan Med Surg 21: 441, 2017.

23. Buzia OD, Fasie V, Mardare N, Diaconu C, Gurau G and Tatu AL: Formulation, preparation, physico-chimical analysis, microbiological peculiarities and therapeutic challenges of extractive solution of Kombucha. Rev Chim 69: 720-724, 2018.

24. Tatu AL and Cristea VC: Unilateral blepharitis with fine follicular scaling. J Cutan Med Surg 21: 442, 2017.

25. Irimie M, Oanţă A, Irimie CA, Fekete LG, Minea DI and Pascu A: Cardiovascular risk factors in patients with chronic plaque psoriasis: A case-control study on the Brasov County population. Acta Dermatovenerol Croat 23: 28-35, 2015.

26. Nwabudike LC and Tatu AL: Using complementary and alternative medicine for the treatment of psoriasis: A step in the right direction. JAMA Dermatol 155: 636, 2019.

27. Nwabudike LC and Tatu AL: Response to: Murphy EC, Nussbaum D, Prussick R, Friedman AJ. Use of complementary and alternative medicine by patients with psoriasis. J Am Acad Dermatol 81: e105, 2019.

28. Modrigan M, Draganescu M, Condratovici CP, Pavel LL and Condratovici AP: Clinical personality patterns in young adults with HIV nosocomial infection from the region of Southeast Romania Mater Plast 54: 175-179, 2017.

29. Batani A, Brănișteanu DE, Ilie MA, Boda D, Ianosi S, Ianosi G and Caruntu C: Assessment of dermal papillary and microvascular parameters in psoriasis vulgaris using in vivo reflectance confocal microscopy. Exp Ther Med 15: 1241-1246, 2018. 